28

\title{
Deploying IN Services in a Mobile Environment
}

\author{
D. Haran
}

Comverse Network Systems

170 Crossways Park Drive, Woodbury, New York 11797 USA

Tel: (516) 677-7200, Fax: (516) 677-7355

E-maildanny_haran@comverse.com

\begin{abstract}
Introducing new IN services into mobile networks presents new challenges with respect to the integration of IN Service Nodes into a mobile environment. This paper examines the issues which are involved in routing calls to and from the Service Node, for both Originating and Terminating IN services, in centralised and distributed system configurations. The paper highlights the inherent difficulties associated with subscriber mobility, and present solutions for the various cases.
\end{abstract}

\section{Keywords}

Service Node, SN, Mobile Networks, IN Services 


\section{INTRODUCTION}

This paper presents a method by which IN services can be seamlessly deployed in a mobile environment, through a Service Node (SN) solution. It introduces the difficulties in getting the calls to the SN, on one hand, and reaching the Mobile Station (MS), on the other hand, without requiring software changes in the network components. Finally, the paper presents possible solutions available with the TRILOGUE INfinity IP/SN.

\section{IN SERVICES ON A SERVICE NODE}

Most subscriber value-added services, which are referred to as IN services, have one common characteristic - they are all "calling services". In other words, they deal with making or receiving telephone calls in a way that brings an added value to the subscriber. The service can affect the way the call is initiated or terminated, how it is billed, etc.

Typical examples for terminating IN services are Personal Number Service and Call Screening. Common originating IN services are Pre-Paid Service, Voice Activated Dialing and Virtual Private Network.

Such IN services can be implemented in several ways, regardless of whether the network has full IN infrastructure or not. In a pre-IN network, IN services are often deployed on a Service Node, which provides all the necessary ingredients, including service logic, switching and voice resources. A self contained SN, such as the TRILOGUE INfinity IP/SN, can deploy IN services even if the telephone network is not yet IN compliant.

When deploying IN services on an SN, all calls must flow through the SN, at least for the call setup phase. The network switches need a way to get calls to the SN, and the SN must be able to deliver the calls to their final destination. This raises several interesting issues, especially in a mobile environment, where mobile subscribers may be "connected" to any of the network switches, depending on their current location.

\section{CALL PROCESSING IN A MOBILE NETWORK}

\subsection{Mobile Network Architecture}

In order to discuss the issues of using an SN in a mobile network, it is important to fully understand the way regular calls are processed and routed by the network, and introduce some of the key concepts and entities used in mobile networks. 
The following schematic diagram shows the basic components of a mobile network, with two switches (MSCs - Mobile Switching Centers), and a mobile subscriber connected to one of the switches through a base station.

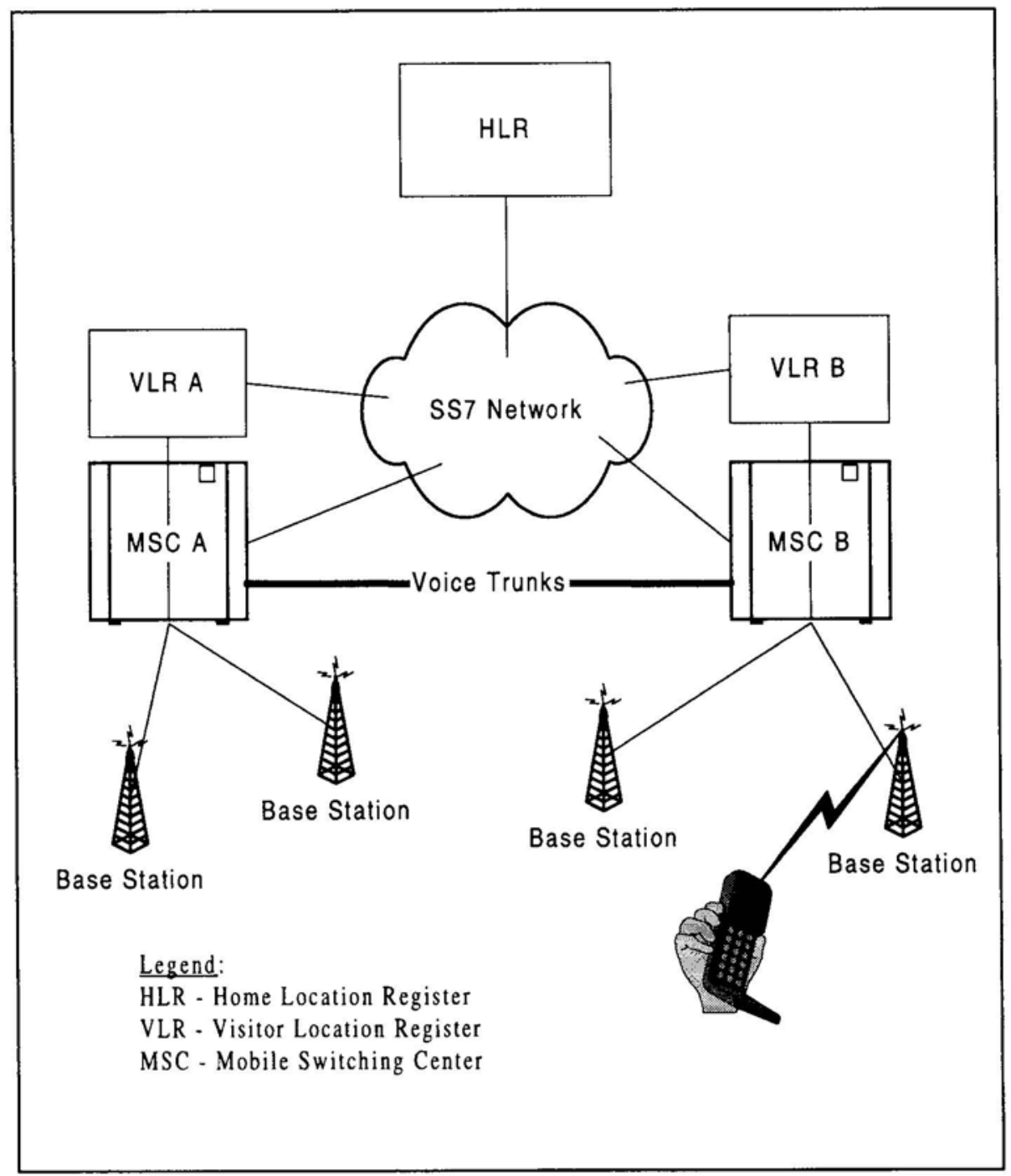

Figure 1 - Mobile Network Entities

The diagram is very schematic, and does not show other systems used for management, authentication, etc. The following systems are shown:

- MSC - Mobile Switching Center. This is the switch within the mobile network. Two MSCs are shown, but a network can contain any number of 
MSCs, depending on its size and geographical disparity. Some of the MSCs may be also used as gateways to the fixed network.

- Base Stations. These are the cellular stations, which handle the wireless communication with the mobile subscribers. Each base station can represent one or multiple cells in the cellular network. In the diagram, only two are shown for each MSC; in reality, one MSC may be connected to dozens or even hundreds of base stations, depending on the area which must be covered by the MSC, cell sizes, etc.

- HLR - Home Location Register. A centralized database of mobile subscribers. One or several HLRs may be used in one mobile network. The HLR stores the subscribers profile, for example their call forwarding options. Most important, the HLR keeps track of the current location of each mobile subscriber, that is, through which MSC the subscriber can be reached.

- VLR - Visitor Location Register. This is a local database for each MSC, where all the mobile subscribers who are currently reachable through the MSC are listed. Whenever a mobile subscriber turns on his handset, or moves into range of one of the MSC base stations, he is registered in the VLR. In addition, the VLR queries the HLR for the subscriber profile, for future use.

Obviously, the main difference between a fixed network and a mobile network, is the mobility of the subscribers. Unlike fixed network subscribers, which are permanently associated with a specific Central Office switch, a mobile subscriber may move around (roam) between areas covered by different MSCs. So the process of routing a call to a mobile subscriber is more complex, and requires knowing (or finding out) through which MSC the subscriber can be reached at any time.

Before describing the process of calling a mobile subscriber, two additional terms need to be defined. The specific terms used are taken from the GSM network, and may have other names in other network types.

- MSISDN (Mobile Station ISDN) Number- This is, simply, the mobile subscriber telephone number. It can contain up to 16 digits (including the area code), and varies in length depending on the home country numbering plan. The MSISDN number is associated with the mobile handset, or, in GSM, the SIM card. It is not changed when the subscriber moves from one MSC to another.

- MSRN (Mobile Subscriber Roaming Number). This is a temporary number assigned to each visiting subscriber by the VLR, which is associated with each MSC. The MSRN is uniquely associated with an MSC/VLR, and is used for routing calls within the network. The idea is to allow the network switches use conventional call switching techniques, which are based on analyzing the Dialed Number. While the MSISDN number cannot be used that way (as it does not imply anything on the target MSC, where the subscriber can be found), the MSRN can be analyzed for routing purposes, since it is linked to 
the visiting MSC/VLR, not with the subscriber. The allocation of the MSRN, and the association between the MSRN and the MSISDN numbers is local to each VLR.

\subsection{Routing Call to Mobile Subscribers}

The following diagram shows the messages exchanged between the various network entities in order to get a call to a Mobile Station (MS), who happens to be in an area covered by MSC B (refer to Fig. 1). The call originates in MSC A, which is referred to as the Gateway MSC (GMSC) for that call. MSC B is called the Visiting MSC (VMSC). The call could have been initiated by another mobile subscriber registered on MSC A, or from a fixed network telephone, assuming that MSC A is also the PSTN gateway. The base station, where the MS is actually connected, and the MS itself are not shown, for simplicity.

The diagram shows two scenarios. The first one takes place when the MS registers at MSC B, for instance after turning on the handset. After establishing a radio connection with one of MSC B's base stations, the MS is registered at VLR $B$, which also notifies the HLR of the new location of the MS. The HLR, in response, will send several fields of the MS profile back to VLR B, where they will be later used if necessary.

The second scenario shows a call for the MS, which arrives at MSC A. The origin of the call is not relevant for the search of the MS. MSC A issues a query to the HLR, asking for an MSRN of the MS. The HLR, knowing that the MS is visiting MSC B, will prompt VLR B for that MSRN, and will return that MSRN to MSC A. Now, MSC A can apply standard routing techniques, based on the MSRN, to select the proper trunk group leading to MSC B, and finally to the MS.

The diagram does not try to follow the call setup all the way through. There may be many different scenarios, depending on whether the MS was busy, or did not answer, on forwarding options, etc. If, for instance, the call needs to be forwarded to another MSISDN number, then MSC B will start the process all over again, so it can route the call to its final destination. 


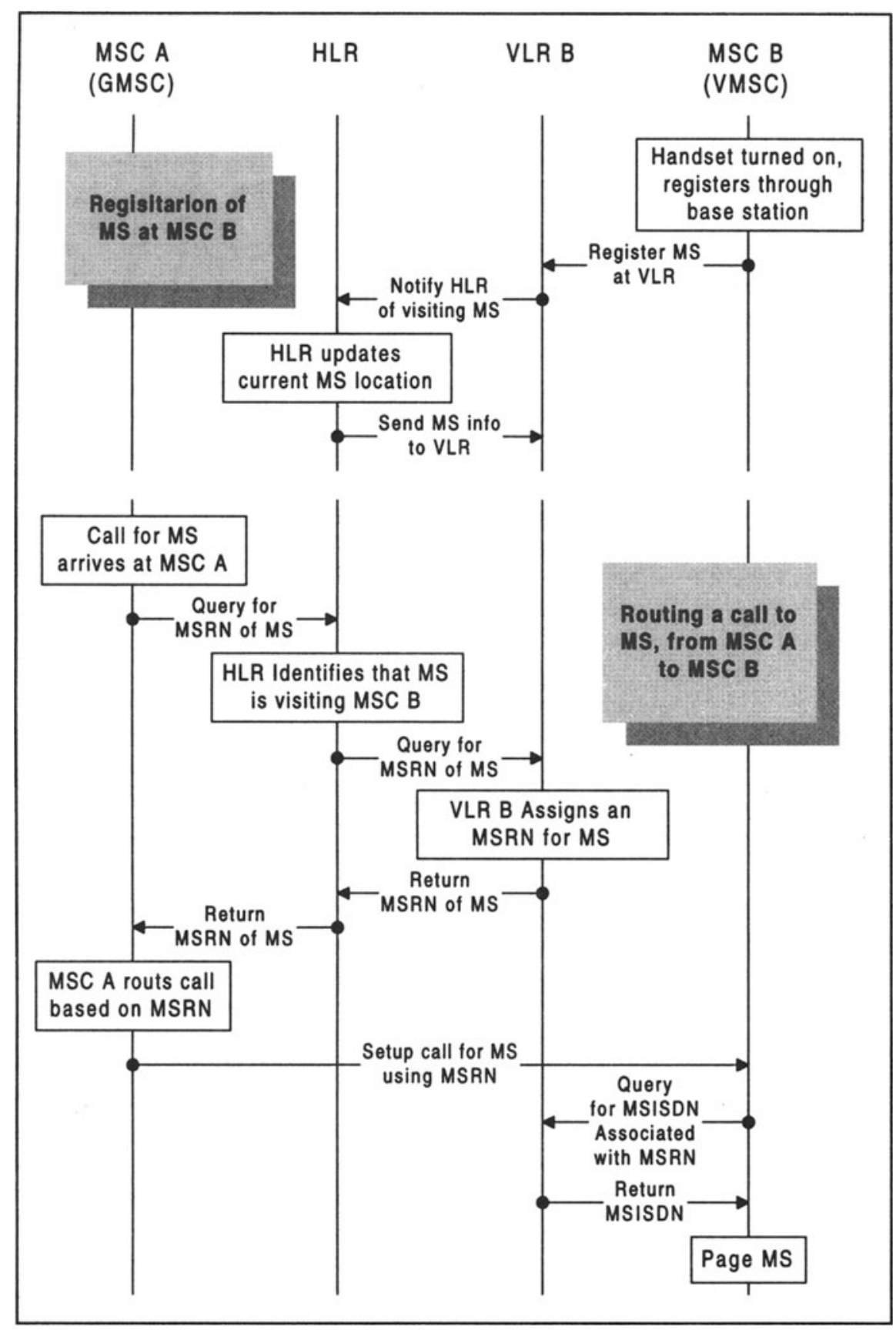

Figure 2 - Routing Calls to a Mobile Subscriber 


\subsection{Service Nodes - the Additional Complexity}

The problems and issues related to deploying service nodes in a mobile network, which are the main topic of this paper, can be attributed to several factors:

- Some services require that all originating or terminating calls for particular subscribers are routed through a service node. MSCs were not built to selectively route calls to a destination other than the dialed number itself. Deciding how to handle each call is a complex task.

- If more than one $S N$ is involved, then the issue of which $S N$ should be selected to provide a specific service for a given subscriber could also be problematic. The HLR was not designed to provide routing information for anything but mobile subscribers, so if MSCs need routing assistance for locating the proper $\mathrm{SN}$, there may be a need in another HLR-like solution for that purpose.

- Some calls directed to an MSISDN number must reach an SN. Other calls, especially those generated by the $\mathrm{SN}$, need to get to the mobile handset. This could result in loops, and may require significant attention to accomplish.

- Originating service calls may present special problems, as they require processing based on the calling party number, which is, again, a task MSCs are not optimized to handle.

The following sections deal in greater detail with these and other issues, and present the solutions, which are available with the Comverse TRILOGUE INfinity IP/SN system.

\section{GETTING CALLS TO THE SERVICE NODE}

In order for the network switches to route calls to the $\mathrm{SN}$, a three-step process is required:

- Identifying that a particular call needs to be delivered to the SN. This is different for originating and terminating services.

- Translating the Dialed Number (DN) of the call to a value can be routed to the SN. The new DN may be a special prefix followed by the subscriber MSISDN number. This step may not be required, if the call was made to a special access code.

- Routing the call to the SN, using standard call routing techniques. This is possible if the previous stage set the $\mathrm{DN}$ to a value which leads to a proper routing tree, which is associated with the $\mathrm{SN}$. 


\subsection{Originating Services}

Originating IN services include Pre-Paid Services (PPS) and Virtual Private Network (VPN). In these services, each call originated by the mobile subscriber must, first of all, get to the SN for processing. Other services, such as Voice Activated Dialing (VAD) require that the subscriber dial some short code in order to access the service.

Whenever the subscriber tries to make an outgoing call, the call is processed by the local MSC covering the area where the subscriber is presently situated. This MSC is known as the Visiting MSC (VMSC) for the mobile subscriber.

In order to identify that this call must be sent to the SN, the VMSC must route the call based on the identity of the caller (the "A number"), which in this case is represented by the subscriber's MSISDN number. Clearly, the dialed number (the "B number") cannot be used for routing to the SN in services such as PPS or VPN, since any number may be dialed.

The VMSC may decide that a call needs to be processed by the $\mathrm{SN}$ in one of several ways:

- Based on the A number, if it is within a predefined number range. This requires that all the telephone numbers associated with a particular service form one or several contiguous number blocks. This requirement is often not acceptable for network operators, since it means that the mobile subscriber has to change his number when subscribing to a new service. In addition, the subscriber is also limited in the number of services he can subscribe to, due to potentially conflicting number ranges.

- Through an HLR interrogation operation, the VMSC can query if the given subscriber has a predefined flag set, representing the IN originating service. If the flag is set, the call needs to be delivered to the SN. The query makes use of the Send Routing Information (SRI) MAP message. Note that this solution requires that a free field is available for that purpose in the HLR subscriber profile.

- Based on the dialed number, if it is equal to a specific access number to that service. That method may be used in VAD, but is not pertinent for VPN and PPS.

Note that this processing only relates to calls, which are originated at the VMSC, directly from the mobile handset. Any other calls, coming over other trunk groups, from other switches or networks, are not subject to this processing.

\subsection{Terminating Services}

In terminating IN services, such as Personal Number Service (PNS), the MSC decides whether a call needs to be transferred to the $\mathrm{SN}$ based on the dialed 
number (the "B number"). The calling number is not relevant, since the call may be originated from any telephone, mobile or not.

The MSC may use one of the following methods to make the decision:

- If the dialed number is within a predefined number block, which is all dedicated to the service. This may not always be applicable, since in many cases mobile subscribers should be able to subscribe to the service without having to change their number.

- Using HLR interrogation, and expecting a special flag in a reserved field of the subscriber profile.

\subsection{Routing the Call to the Service Node}

Once the decision has been made to transfer the call to the $\mathrm{SN}$, both for originating and terminating services, then routing the call to the $\mathrm{SN}$ is done using regular switch routing techniques, as explained below.

The call is sent to the SN either through a dedicated trunk group, or by changing the dialed number to a specific predefined number which is associated with the SN and the specific service. The network MSCs are programmed to route this number to the SN, even if they do not have a direct connection to it.

Regardless of the method used, it is crucial that the $\mathrm{SN}$ receives both the original A and B numbers, so it can process the call properly. Also, there must be an indication of the exact service being requested. This may be implied by the predefined number used to access the SN, or through any other IAM message field. However, if the network cannot identify (or cannot deliver) the exact service required, the $\mathrm{SN}$ can still identify the service based on the subscriber profile stored in the SN. Alternatively, the SN can query a remote subscriber profile, for instance, through HLR interrogation.

Note that in a distributed environment, where multiple service nodes provide the service, routing the call to the right $\mathrm{SN}$ may be quite complex. The issue of distributed solutions is discussed in section 7 below.

\section{CALLING THE MOBILE SUBSCRIBER}

Once the call has reached the SN, it is processed according to the service, the subscriber profile and the call parameters. Often, the SN needs to make an outgoing call, resembling the original call in many ways. Getting this call through the network is the tricky part of deploying IN services in mobile networks.

For instance, in a PNS scenario, the SN may wish to deliver the incoming call to the mobile subscriber, based on the PNS subscriber profile. The problem is that the PNS number is usually the same number as the mobile number (the MSISDN number), so unless some measures are taken, the network may bounce that call back to the SN, creating infinite loops. There must be a way for the mobile 
network to differentiate calls coming from the $\mathrm{SN}$ and calls coming from any other origin, even if they are trying to reach the same number.

There are two ways in which the $\mathrm{SN}$ and the mobile network can handle the situation, avoiding potential loops:

- By initiating all calls from the SN to the MSC on a dedicated trunk group, and thus guiding the switch to handle the call arriving on this trunk group in a different manner than what is described above. Hence, for such calls, the MSC does not check if the dialed number refers to any terminating service such as PNS, but rather, handles the call in the standard way, just like any call directed to a mobile subscriber. One problem with this approach is that it may require dividing the trunk group leading from the SN to the MSC into several smaller trunk groups, each dedicated for a specific service and use.

- By issuing calls from the SN to the network using an MSRN number rather than the MSISDN number. The TRILOGUE INfinity IP/SN can interrogate the HLR, using the SRI message, and route the call based on the returned MSRN number. The call will be handled in a totally different manner because now it is routed by the network based on the MSRN and not the MSISDN number, effectively eliminating infinite loops.

The second solution provides a smoother integration of the $\mathrm{SN}$ with the mobile network, by not imposing any dedicated trunk groups from the $\mathrm{SN}$ to one or several MSCs. It utilizes the TRILOGUE INfinity IP/SN HLR Interrogation capability, making the $\mathrm{SN}$ tightly integrated into the mobile network.

\section{COMPLETING ORIGINATING SERVICE CALLS}

In an IN originating service, such as Pre-Paid Service (PPS), the SN issues the outgoing call "on behalf" of the mobile subscriber, either to another mobile subscriber or to a PSTN number. Here, in order to maintain features such as CLI display, the $\mathrm{SN}$ needs to initiate the call with the same $\mathrm{A}$ number as the original call, which is the PPS subscriber MSISDN number. Here also, there is a potential problem of infinite loops, if the network cannot differentiate between the call from the $\mathrm{SN}$ and the call coming from the mobile handset. The potential problem is that the MSC will look at the A number and find out that it relates to a PPS subscriber, and thus route the call back to the $\mathrm{SN}$.

There are two ways, in which the SN outgoing call can be distinguished from a call originating from the mobile handset:

- The best way is to have the switches only refer to the A number on calls coming directly from mobile subscribers, that is, from trunk groups connecting the MSC to its base stations. That way, only the originating MSC will ever look at the A number and will check if this call has to be transferred to the SN. Other MSCs, which may be transferring the call to the SN will not do any A 
number processing. Similarly, calls originated at the $\mathrm{SN}$ will not be subject to any such IN originating service processing.

- If, for some reason, the above method cannot be used, there is always the possibility of using another A number when making the outgoing call from the SN. That is, the SN will use its own predefined number in the Calling Party Number (CGPN) field. This special number is not configured on the network as a subscriber to an IN originating service, hence it is not subject to any originating service processing, and the call is handled and routed as a regular telephone call.

The one clear disadvantage of this method is the fact that the real subscriber MSISDN number is not used as the Calling Party Number, which can affect other value added services normally available to the recipient of the call, such as CLI Display, Call Back, or any CLI-based Screening.

\section{DISTRIBUTED MULTI-NODE NETWORKS}

The issue of routing calls to an $\mathrm{SN}$ is even more complicated in a distributed environment, where there are multiple service nodes performing one or several services. Under these conditions, the MSC has to find out, on a per call basis, the correct service node identity, depending both on the subscriber ID and the service requested.

The problem of routing calls to multiple systems exists even in non-calling services, such as Voice Mail (VM). There are several cases where a distributed multi-node solution is deployed in a network:

- The network is very large, and a single platform cannot service all the subscribers.

- The network is geographically dispersed, and using several local platforms is more cost-effective than using one large central system.

- Existing systems cannot be expanded to provide additional capacity, and another system, sometimes of a new vendor, is added to service new subscribers.

\subsection{Voice Mail Call Routing}

A special case, which can use the existing mobile network forwarding mechanism to do routing, is for Voice Mail (VM) message deposit calls, where calls are conditionally forwarded to the VM system based on busy or no-answer states.

The forwarding number is temporarily stored at the VLR, after it has been downloaded from the HLR when the subscriber registered at the VMSC. Hence, when the VMSC fails to deliver the call to the mobile subscriber, it uses the forwarding number to send the call to the VM system. Potentially, the forwarding number can be individually specified for each subscriber, so that each subscriber 
can be assigned a forwarding number corresponding to the VM system where his mailbox is stored.

In most networks, using the busy/no-answer forwarding numbers for VM usually results in restricting any subscriber self modification of these numbers. Hence, when subscribing to VM, subscribers lose their busy/no-answer forwarding options. Only unconditional call forwarding can be used by VM subscribers.

Unlike message deposit, Message retrieval may be handled in one of two ways:

- The subscriber may call his own number, resulting in a busy condition, hence the call is forwarded to the VM system in the same way a guest call is. In fact, some VM systems cannot even tell that this is a subscriber message retrieval call, until the subscriber performs a login procedure, e.g., by pressing “*” followed by his password. More sophisticated VM systems, such as the TRILOGUE INfinity, can identify that the calling number is identical to the subscriber ID number, and start a message retrieval session with no explicit login process.

- The common solution is for the subscriber to dial an access short code to enter his mailbox. The VMSC should identify this short code and transfer the call to the correct VM system. In this case, since the busy/no-answer states do not apply, no forwarding takes place, and the VMSC must use "A Number" routing, as described above. Getting to the right VM system is more complex, since the forwarding number cannot be used.

Since most mobile network operators require that a short code be used to retrieve VM messages, the call forwarding mechanism cannot provide a satisfactory solution for accessing multiple VM systems. Alternative methods for getting calls to the right VM system are described below.

\subsection{Getting to the Right Node}

In most cases where multiple systems are deployed, doing either Voice Mail or other services, the easy and straightforward way to find the right node for each subscriber, is to use predefined number ranges. Under this scheme, every platform is associated with a predefined MSISDN number range (e.g., 10,000 numbers). Every call related to a subscriber whose number falls within this range is routed to the specific node.

Actual routing usually requires the addition of a specific prefix to the MSISDN number, so that the call is routed to the appropriate $\mathrm{SN}$, and not to the actual subscriber. Different prefixes are used for different service node types (e.g., one for VM and another for PNS). For message deposits, the routing prefixes are usually part of the HLR call forwarding fields, which contain both the prefix and the MSISDN number. For message retrieval using a short code, the prefix is inserted to the Called Party Number by the routing tree used for that short code, depending on the MSISDN number range. 
The advantages of the number range method is its simplicity, and its suitability for switch routing. This is, essentially, the way all calls are routed. It is also local to the switch, requiring no more than the correct setting of the routing tables, and use of standard GSM call forwarding mechanisms.

Yet, that simplicity is also the drawback of the number range solution. It allows no flexibility at the single subscriber level. It is not possible to allocate subscribers to platforms based on any other parameter except their MSISDN number. Worse than that, it is impossible to move a single subscriber from one platform to another without changing its MSISDN number, which is generally unacceptable.

The ability to allocate single subscribers to specific service nodes is very important to network operators. The issue is often raised when the operator introduces a new platform, with additional service features, and wishes to allow subscribers to upgrade their service to include the new features without changing their number. Another case is when an old system reaches its capacity limit, and new subscribers must be allocated to a new one, regardless of their MSISDN number.

The only way an MSC can tell where to route a call on a per-subscriber basis (except for call forwarding), is by issuing a query to an external database, and receiving the correct Service Node ID in the response message. The query parameter must include the subscriber MSISDN number as well as an indication of the required service, since separate services may be deployed on different service nodes.

A routing database may be queried by the MSC in one of several ways, explained below. Note, this database may be the actual network HLR or any other networkwide "SCP-like" entity, which the switches query when they need the routing information.

\subsubsection{HLR Interrogation}

The network HLR cannot always be used for getting the proper SN routing information, since in many networks it will only accept simple MSISDN-based routing queries, which result in the MSRN of the mobile subscriber (handset) - and not any service node routing information. In other networks, the MSISDN number contains a special part (e.g., two digits), which can be used to identify a specific service query, using the regular Send Routing Information (SRI) MAP message.

If the MSC can indeed query the HLR for the MSRN by providing a special service query indicator, the HLR interprets this query as related to the service node rather than the mobile handset. The HLR can then request the MSRN from a designated TRILOGUE INfinity system, which has the whole routing database, and functions as a pseudo VLR for that purpose. Just like the HLR queries regular VLRs for mobile subscribers MSRN, using the PRN (Provide Routing Number) MAP message, the same is done regarding the TRILOGUE INfinity "VLR". 
Note that the TRILOGUE INfinity "VLR" can hold the routing information for every subscriber in the network, including those subscribers which have their mailboxes on other VM systems, of any type (TRILOGUE INfinity or other).

\subsubsection{MAP Query}

If the HLR cannot be used for this task, because of inherent limitations, then another network-wide database must be deployed to serve that need.

The MSC may issue a MAP SRI message to that database, with the subscriber MSISDN number and an indication of the required service (e.g., as a prefix) in the $B$ number field. The return value may be an MSRN used to route the call to the $\mathrm{SN}$, or, in fact, any number which can be used by the switch for routing.

Note that the SRI message may be inappropriate in the case of A number routing, since several types of MSCs cannot place the A number in the "B number" field of the SRI message. So, for instance, if a Voice Mail subscriber calls to retrieve his messages using a fixed short code such as "*123", then the SRI message can only have the original B number, that is "*123", which makes the query useless.

\subsubsection{INAP Query}

When MAP messages cannot be used due to limitations of the SRI message and how it is used, a more advanced INAP query mechanism may be applied in a similar way.

The MSC issues an InitialDP INAP message, which contains both the A \& B numbers, as well as the service key field, which identifies the service to which the query is related.

The database looks at the subscriber's profile and responses with the Connect INAP message. The Connect message includes the Destination Routing Address field, which is usually an MSRN, which uniquely identifies the appropriate SN.

\subsection{Distributed INfinity - The "No Routing" Solution}

The TRILOGUE INfinity distributed architecture offers another innovative solution for the problem of routing calls to several service nodes.

With Distributed INfinity (DIN), all the TRILOGUE INfinity nodes are connected via high bandwidth data communication links, creating a service node cluster. Each call, reaching any $S N$, may be handled by that $S N$, even if the subscriber profile or mailbox resides on another TRILOGUE INfinity system.

For VM, a distributed session is established between the local system front-end unit (MMU) and the subscriber home system back-end unit (MSU). Thus, voice may be recorded or played over the distributed data network, allowing ubiquitous access to any mailbox from any node. 
For IN services, the situation is normally simpler, since all that is required is for the local service node to query the home service node once for the subscriber profile, so it can serve this subscriber.

The following diagram shows three TRILOGUE INfinity systems, interconnected via an inter-site network, connected to three network MSCs.

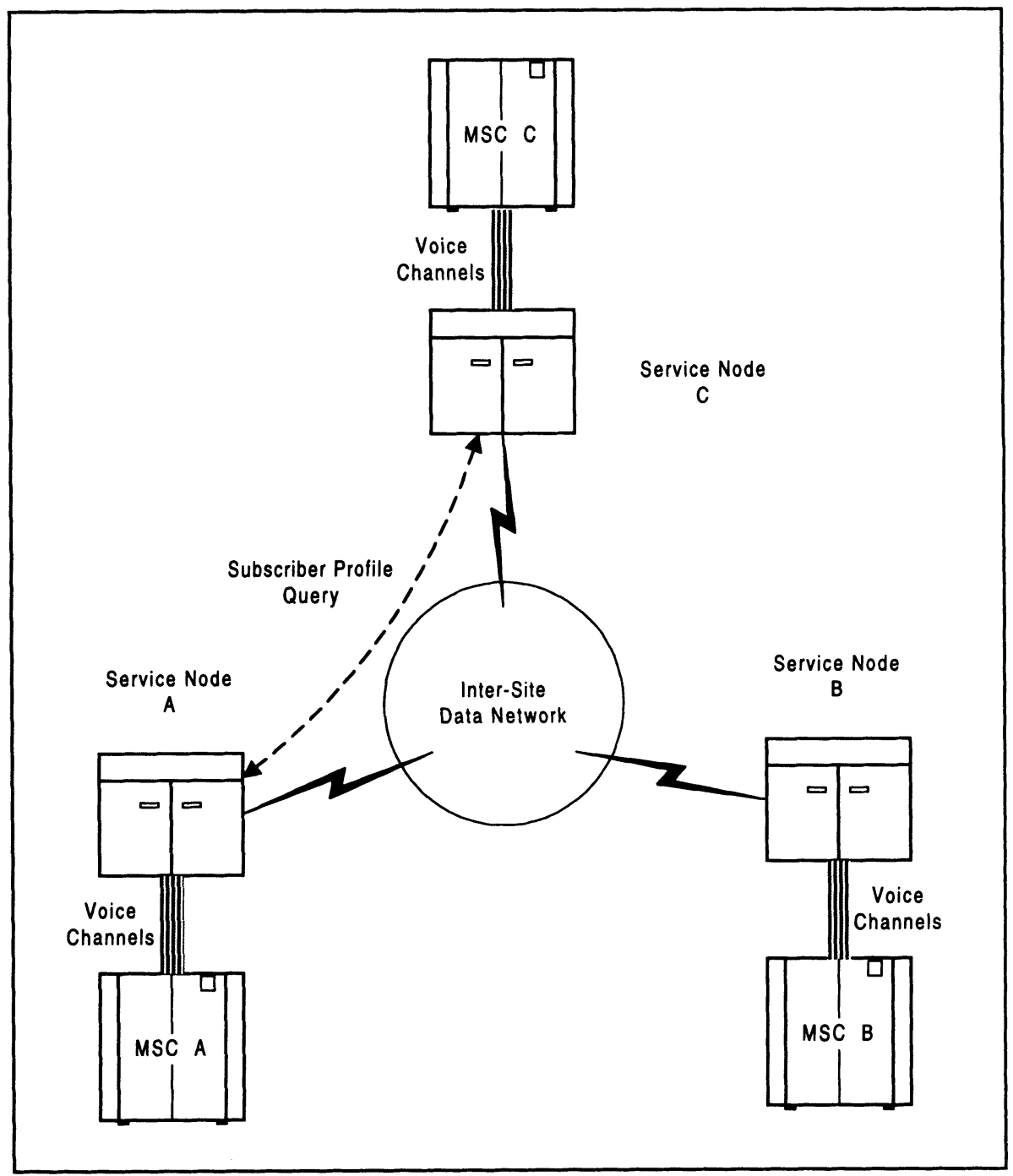

Figure 3 - Distributed INfinity Configuration

A typical scenario would be a call reaching MSC "A", which needs to be processed by a specific IN service. Once MSC " $A$ " identifies that the call needs to be routed to an SN, it always forwards the call to the closest SN, which is SN "A". 
If the subscriber profile resides on SN "A", then the call is processed locally. However, if the profile is not found in $\mathrm{SN}$ " $\mathrm{A}$ " database, the SN performs the following steps:

- SN " $A$ " issues an "Address Resolution" query to the DIN network, looking for the $\mathrm{SN}$ which holds the subscriber record related to the service being provided.

- Once a response is received (assuming it is SN " $C$ "), " $A$ " queries " $C$ " for the subscriber record. In fact, only the subscriber profile fields which are necessary for processing the call are sent back in response.

- SN "A" continues to process the call, based on the retrieved profile.

- Once the call is completed, SN "A" may need to update "C" with respect to possible changes in the profile (e.g., decrement the remaining balance in a PrePaid service).

Note that Voice Mail calls are handled differently, since a distributed voice session is established between the local system and the subscriber "home" system.

Note that by having a Distributed INfinity solution, the multi-node routing issue is, in fact, eliminated. As shown in the figure, each MSC may be connected to one of the nodes, and route all calls to that node, knowing they can be processed by that node regardless of whether the subscriber really has his account on that node, or not. In other words, each MSC is only aware on one service node, where it routes all calls where some service is required.

Clearly, such a "no routing" multi-node solution is only possible for a homogeneous architecture, where only TRILOGUE INfinity systems are deployed, and where they are all connected in one Distributed INfinity cluster. 